\title{
DIELECTRIC AND OPTICAL PROPERTIES OF EUROPIUM OXIDE FILMS
}

\author{
M. K. JAYARAJ AND C. P. G. VALLABHAN \\ Department of Physics, Cochin University of Science and Technology, Cochin 682022 (India) \\ (Received February 15, 1989; accepted May 16, 1989)
}

The dielectric properties of vacuum-deposited europium oxide films have been investigated in the frequency range from $1 \mathrm{kHz}$ to $1 \mathrm{MHz}$ at various temperatures $(300-543 \mathrm{~K})$. The dielectric constant is found to depend on film thickness and it attains a constant value beyond $1000 \AA$. Films deposited at higher substrate temperatures (above $423 \mathrm{~K}$ ) exhibit improved dielectric properties owing to the recovery of stoichiometry. The frequency variation of the loss factor exhibits a minimum which increases with rise in temperature. The breakdown field strength (about $10^{6} \mathrm{~V} \mathrm{~cm}^{-1}$ ) is found to be thickness dependent and it varies in accordance with the Forlani-Minnaja relation. The films exhibit ohmic conduction with an activation energy of $0.86 \mathrm{eV}$ at low electric fields but at higher fields the conductivity becomes space charge limited. X-ray studies show that the films are amorphous in nature. The a.c. conductivity is proportional to $\omega$ at low frequency, whereas a square law dependence is observed at higher frequencies. The optical constants $n, \alpha$ and $k$ and optical band gap are calculated from the UV-visible-near-IR spectra.

\section{INTRODUCTION}

Rare earth oxide films have been studied previously ${ }^{1-5}$ with a view to their use in thin film capacitors in which the required degree of perfection varies according to the specific application. The high dielectric constant and low dielectric loss are generally desirable for low volume capacitors. The variations in these parameters with temperature, frequency, film thickness and applied field are also of such importance as to merit serious consideration in many situations. While a low dielectric constant is preferable for insulating films, dielectric constants in the range $7-30$ and relatively low dissipation factors of $0.55 \%-2.5 \%$ are reported for oxides of lanthanum ${ }^{6}$, cerium ${ }^{7}$, neodymium ${ }^{4}$, dysprosium ${ }^{3}$ and erbium ${ }^{1}$. Studies on $\mathrm{Eu}_{2} \mathrm{O}_{3}{ }^{5}$, $\mathrm{Pr}_{2} \mathrm{O}_{3}{ }^{8}, \mathrm{Nd}_{2} \mathrm{O}_{3}{ }^{4}$ and $\mathrm{Dy}_{2} \mathrm{O}_{3}{ }^{3}$ thin films, which form useful capacitors, revealed a thickness-dependent dielectric constant. The dielectric properties of rare earth oxide films are found to change with annealing or aging and they required an annealing time of about $200 \mathrm{~h}$ to have stable dielectric properties ${ }^{5}$. In this paper we report the dielectric and optical properties of evaporated $\mathrm{Eu}_{2} \mathrm{O}_{3}$ films which exhibit improved dielectric properties when prepared with higher substrate temperatures. 


\section{EXPERIMENTAL DETAILS}

High purity $(99.99 \%) \mathrm{Eu}_{2} \mathrm{O}_{3}$ pellets were vacuum deposited from tungsten baskets onto thoroughly cleaned glass substrates maintained at a temperature of $150{ }^{\circ} \mathrm{C}$. Vacuum-evaporated aluminium $(99.99 \%$ ) thick films (about $0.2 \mu \mathrm{m}$ ) form the rear and front electrodes in a metal-insulator-metal structure. The effective area of a capacitor thus fabricated was $0.36 \mathrm{~cm}^{2}$. The dielectric and electrical measurements were carried out under vacuum $\left(10^{-2}\right.$ Torr $)$ conditions. The film thickness was measured by Tolansky's interference method with an accuracy of $\pm 10 \AA$.

The capacitance and loss factor were measured over a range of frequencies $(1 \mathrm{kHz}-1 \mathrm{MHz})$ and temperatures $(300-543 \mathrm{~K})$ for various film thicknesses $(500$ $2500 \AA$ ) using a $0.1 \%$ Universal Bridge and LCZ Bridge (Hewlett-Packard model 4277A). The temperature was monitored using a chromel-alumel thermocouple with an accuracy of $\pm 1^{\circ} \mathrm{C}$. The dielectric constant was evaluated from the capacitance measurement data using the area and thickness of the film. The currentvoltage characteristics and breakdown voltage were measured using an electrometer and a $4 \frac{1}{2}$ digit autoranging multimeter. The optical constants were calculated from the transmittance spectra in the UV-visible-near-IR region obtained using a Hitachi spectrophotometer (model 330).

\section{RESULTS}

\subsection{X-ray diffraction}

X-ray diffraction studies show that the films obtained are essentially amorphous in nature. No indications of crystallinity were observed for films deposited at substrate temperatures of $150-170^{\circ} \mathrm{C}$.

\subsection{Dielectric properties}

Figure 1 shows the variation in dielectric constant $\varepsilon$ and loss factor $\tan \delta$ as a function of $\mathrm{Eu}_{2} \mathrm{O}_{3}$ film thickness. The dielectric constant evaluated from the measured capacitance and dimensions of the film increases with increase in film

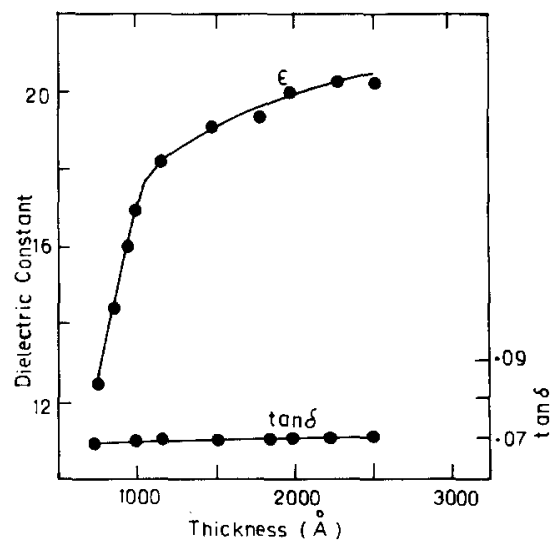

Fig. 1. Variation in dielectric constant $\varepsilon$ and loss factor $\tan \delta$ with dielectric film thickness. 
thickness and attains a steady value of 20.4 for insulator thicknesses greater than $1000 \AA$. The loss factor of 0.07 evidently is independent of film thickness. A similar thickness dependence of $\varepsilon$ has been observed for thin films of other rare earth oxides $^{3-5}$.

Figure 2(a) shows the variation in dielectric constant $\varepsilon$ with frequency at different temperatures for a film thickness of $1200 \AA$. At higher temperatures $\varepsilon$ increases drastically with decrease in frequency. The variation in $\varepsilon$ with frequency is small at room temperature. From the plot of capacitance $v$ s. temperature at different
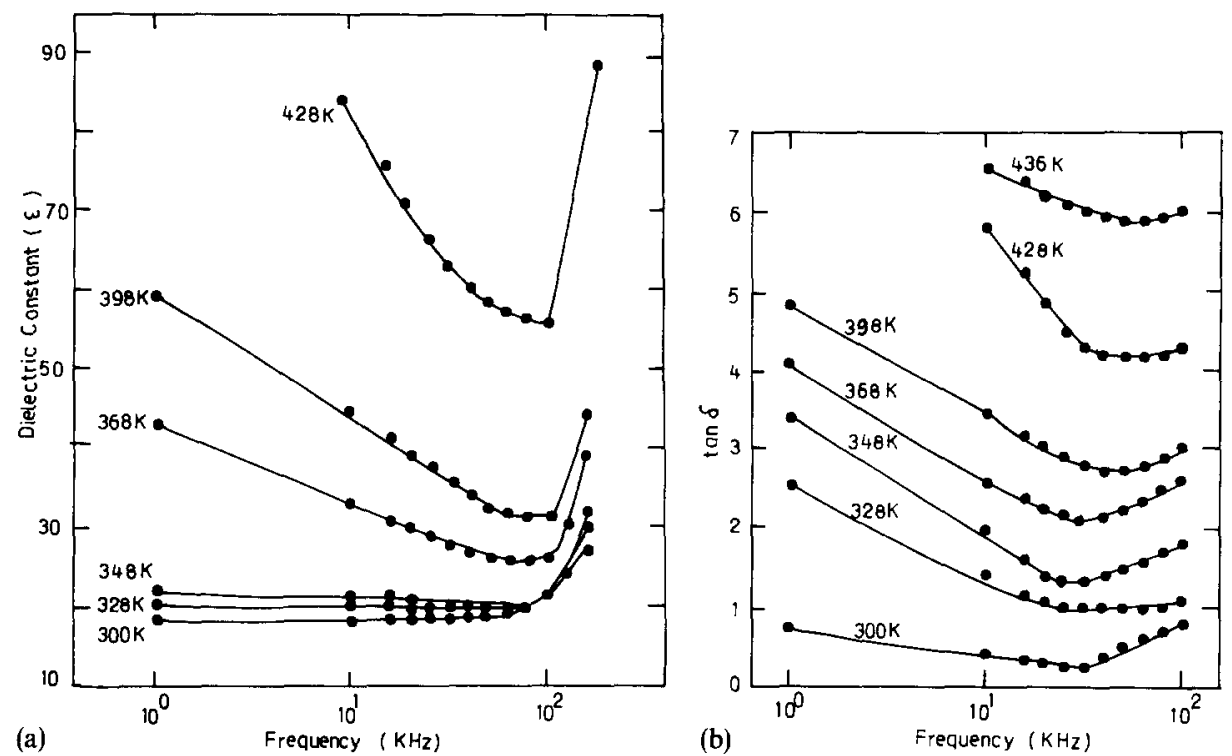

Fig. 2. (a) Variation in dielectric constant $\varepsilon$ with frequency at different temperatures (film thickness, $1200 \AA$ ); (b) variation in $\tan \delta$ with frequency at different temperatures (film thickness, $1200 \AA$ ).

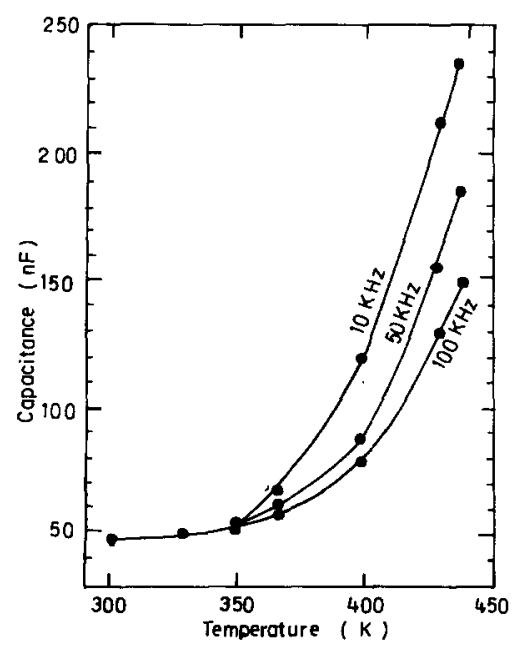

Fig. 3. Capacitance $v s$. temperature at different fixed frequencies (film thickness, $1200 \AA ̊$ ). 
frequencies (Fig. 3) it is observed that below room temperature the plots merge to a single curve and the capacitance varies only slightly when the temperature is low. On extrapolating we obtain the value of static dielectric constant $\varepsilon_{\mathrm{s}}=16.4$. The temperature coefficient of capacitance $\gamma_{c}=(1 / C) \mathrm{d} C / \mathrm{d} t$ was also obtained from the graph, and this has a value of $234 \pm 10 \mathrm{ppm} \mathrm{K}^{-1}$ at room temperature (evaluated at $1 \mathrm{kHz}$ ). This value compares favourably with those reported in the literature $\mathrm{e}^{3,9,10}$ for other well-known dielectric materials. The variation in $\tan \delta$ with frequency at different temperatures is shown in Fig. 2(b). The $\tan \delta$ value decreases with increase in frequency and attains a minimum value which itself decreases at higher temperatures. At low temperatures the decrease in $\tan \delta$ with frequency is less marked.

\subsection{Electrical properties}

From the current-voltage $I-V$ measurements carried out it can be seen that the films exhibit ohmic conduction at low electric fields but at higher fields the conduction becomes space charge limited so that $I \propto V^{2}$. From the conductivity plot $\left(10^{3} / T v s . \ln \sigma ;\right.$ Fig. 4) the activation energy is found to be $0.86 \pm 0.03 \mathrm{eV}$. The space-charge-limited current and low activation energy suggest the presence of shallow traps in the films. Consequently the observed activation energy is less than the expected band gap.

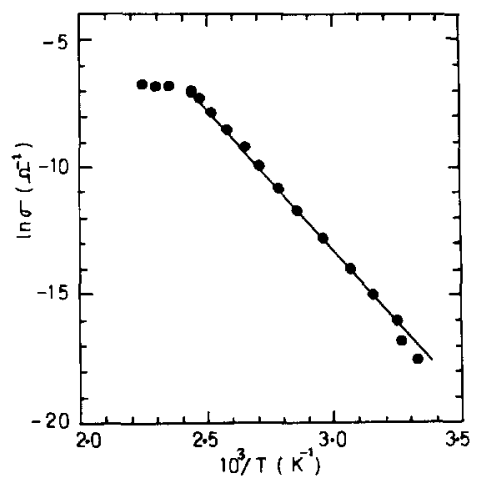

Fig. 4. $\ln \sigma$ vs. $10^{3} / T$.

The dielectric breakdown field strength $F_{\mathrm{b}}$ is of the order of $10^{6} \mathrm{~V} \mathrm{~cm}-1$. The breakdown strength $F_{\mathrm{b}}$ is found to decrease with increase in film thickness. A loglog plot of the dielectric breakdown strength $v$ s. film thickness yields a straight line (Fig. 5) with a negative slope of 0.518 from which it can be found that the breakdown field strength follows the Forlani-Minnaja ${ }^{11}$ relation: $F_{b} \propto d^{-1 / 2}$.

The variation in a.c. conductivity at various temperatures with frequency is shown in Fig. 6. Here the conductivity exhibits two distinct dispersive regions, one below $100 \mathrm{kHz}$ and the other above it. The a.c. conductivity is porportional to $\omega$ in the first region and becomes proportional to $\omega^{1.9}$ in the second region at room temperature. The same trend is also observed at higher temperatures. The second region of the conductivity thus exhibits a square law dependence on frequency. 
From the plot of $\ln \sigma v s 10^{3} / T$ the activation energy is calculated to be $0.13 \pm 0.03 \mathrm{eV}$ in the temperature range $300-365 \mathrm{~K}$ at $10 \mathrm{kHz}$ (Fig. 6).

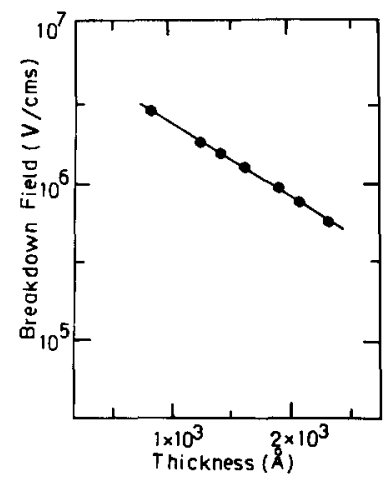

Fig. 5. Breakdown field strength $v$ s. dielectric film thickness.

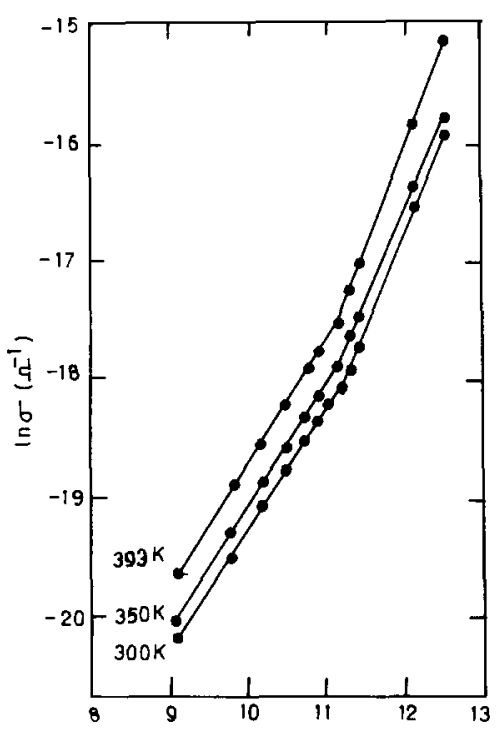

(a)

In $2 \pi t(\mathrm{~Hz})$

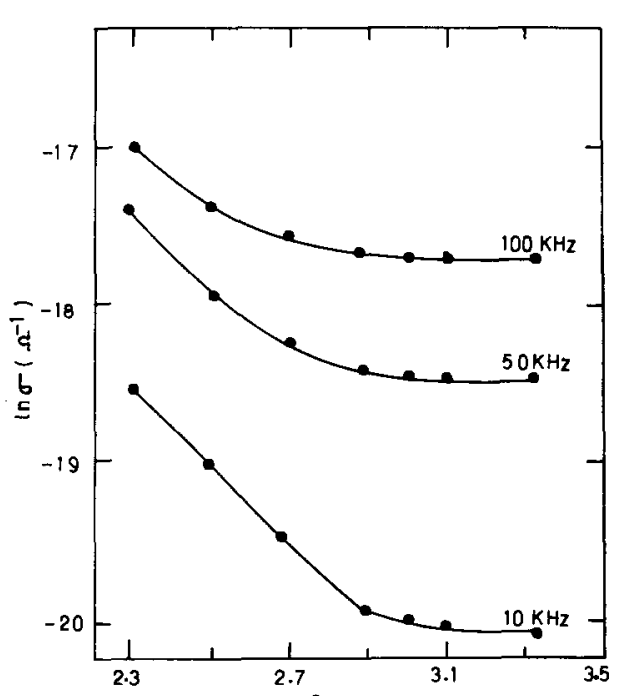

(b)

$10^{3} / T\left(K^{-1}\right)$

Fig. 6. (a) A.c. conductivity $v$ s. frequency at different temperatures (film thickness, $1200 \AA$ ); (b) $\ln \sigma v s$. $10^{3} / T$ at different fixed frequencies (film thickness, $1200 \AA$ ).

\section{OPTICAL PROPERTIES}

To evaluate the optical constants we used the method proposed by Manifacier et al. ${ }^{12}$ which makes use of the fringe pattern of the transmission spectrum of a thin transparent dielectric film surrounded by non-absorbing media. The film can be considered to be bounded by two transparent media with refractive indices $n_{0}$ and $n_{1}$. If we consider a unit amplitude for the incident light (in this case normal 
incidence) and the case of weak absorption where $k^{2} \ll\left(n-n_{0}\right)^{2}$ and $k^{2} \ll\left(n-n_{1}\right)^{2}$, the transmission $T$ is given by

$$
T=\frac{16 n_{0} n_{1} n^{2} \alpha_{1}}{c_{1}{ }^{2}+c_{2}{ }^{2} \alpha_{1}{ }^{2}+2 c_{1} c_{2} \alpha_{1} \cos (4 \pi n t / \lambda)}
$$

where

$$
\begin{aligned}
& c_{1}=\left(n+n_{0}\right)\left(n+n_{1}\right) \text { and } c_{2}=\left(n-n_{0}\right)\left(n_{1}-n\right) \\
& \alpha_{1}=\exp (-4 \pi k t / \lambda)=\exp (-\alpha t)
\end{aligned}
$$

Here $\alpha$ is the absorption coefficient of the thin film and $t$ is its thickness; $k$ and $\lambda$ are the extinction coefficient and the wavelength of the light respectively.

The extreme values of the transmission are given by

$$
\begin{aligned}
& T_{\max }=16 n_{0} n_{1} n^{2} \alpha_{1} /\left(c_{1}+c_{2} \alpha_{1}\right)^{2} \\
& T_{\min }=16 n_{0} n_{1} n^{2} \alpha_{1} /\left(c_{1}-c_{2} \alpha_{1}\right)^{2}
\end{aligned}
$$

Thereby

$$
n=\left\{N+\left(N^{2}-n_{0}{ }^{2} n_{1}{ }^{2}\right)^{1 / 2}\right\}^{1 / 2}
$$

where

$$
N=\frac{n_{0}{ }^{2}+n_{1}{ }^{2}}{2}+2 n_{0} n_{1} \frac{T_{\max }-T_{\min }}{T_{\max } T_{\min }}
$$

Thus $n$ can be determined from knowledge of $T_{\max }, T_{\min }, n_{1}$ and $n_{0}$ at the same wavelength. The accuracy in $n$ is a critical function of the errors in the measured values of $T_{\max }$ and $T_{\min }$. In the best case the precision possible in these measurements is of the order of $1 \%$ giving an overall accuracy of $2 \%$ for the value of $n$. Using eqns. (3) and (4) we can find $\alpha_{1}$ and then eqn. (2) gives the absorption coefficient $\alpha$ and extinction coefficient $k$. Figure 7 shows the variation in $n$ and $k$ with wavelength. The value of the refractive index is found to remain constant (about 1.64) in the visible and IR regions. Outside the region of fundamental absorption, the dispersions of $n$ and $k$ are not very large. The absorption coefficient $\alpha$ and extinction coefficient $k$ evaluated from the transmission data were found to be almost zero for longer wavelengths, i.e. below the fundamental absorption frequency. Figure 8 shows the dependence of absorption coefficient on photon energy. The optical absorption data of $\mathrm{Eu}_{2} \mathrm{O}_{3}$ films were examined for evidence of either indirect or direct transitions in accordance with the theory of Bardeen et al. ${ }^{13}$ For such transitions

$$
\alpha=\frac{A\left(h v-E_{\mathrm{g}}{ }^{\prime} \pm E_{\mathrm{p}}\right)^{r}}{h v}
$$

where $r=3$ for forbidden indirect transitions, $r=2$ for allowed indirect transitions and $E_{\mathrm{g}}{ }^{\prime}$ is the indirect band gap, $E_{\mathrm{p}}$ being absorbed or emitted phonon energy. The plot of $(\alpha h v)^{2} v s$. photon energy $h v$ gives a straight line portion and on extrapolation the intercept gives a band gap of $3.38 \pm 0.1 \mathrm{eV}$. Thus the conclusion may be drawn that the transition leading to fundamental absorption takes place at $3.38 \pm 0.1 \mathrm{eV}$ and the transition is indirect. 


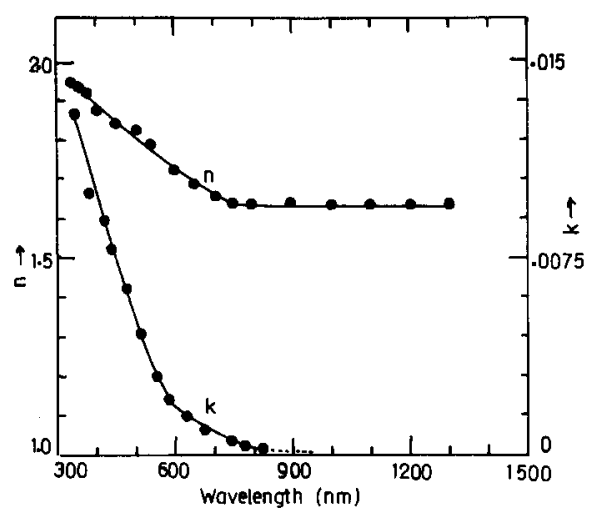

Fig. 7. Variation in refractive index and extinction coefficient of the dielectric film with wavelength.

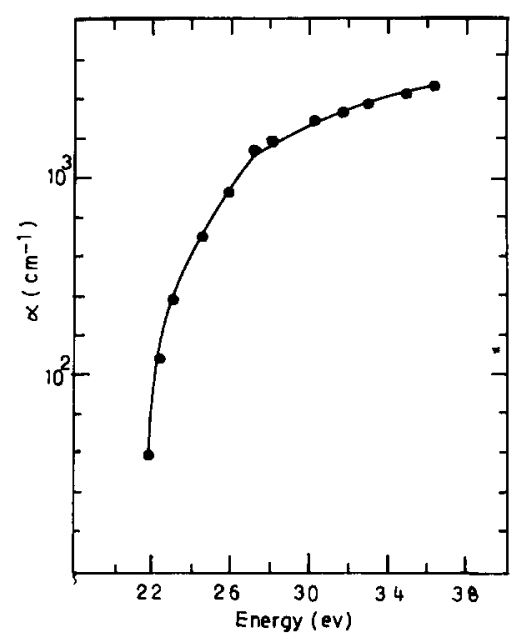

Fig. 8. Absorption coefficient $\alpha$ vs. photon energy $h v$.

\section{DISCUSSION}

The results outlined above give clear indications of the physical behaviour of $\mathrm{Eu}_{2} \mathrm{O}_{3}$ films prepared in the laboratory. The films are essentially amorphous in nature and the X-ray studies reveal no crystallinity for films prepared at higher substrate temperatures. This is fully in accordance with the observation that vacuum-evaporated rare earth oxide films are generally amorphous in nature ${ }^{3-5}$. The observed thickness dependence of the dielectric constant for films of thickness less than $1000 \AA$ is apparently due to defects such as voids, stress and inhomogeneity which are normally present in vacuum-deposited films rather than being due to nonstoichiometry of the deposits caused by excess of oxygen or metal ions. As films become thicker, the density of voids decreases ${ }^{14}$, resulting in higher values of the dielectric constant, which eventually becomes thickness independent. A theoretical 
analysis by Kornyshev et al. ${ }^{15}$ suggests that the observed effect was too large to be ascribed to the non-local nature of the dielectric response alone and the presence of voids and other inhomogeneities contributes to the observed anomaly in rare earth oxide films. The observed dependence of $\varepsilon$ and $\tan \delta$ on frequency and temperature may be accounted for by considering the equivalent circuit proposed by Goswami and Goswami ${ }^{16}$. This comprises an inherent capacitive element $C_{\mathrm{i}}$ unaffected by frequency and temperature, a discrete resistance element $R$ which is assumed to be temperature dependent and in parallel with $C_{\mathrm{i}}$ and a series resistance $r$ due to lead resistance. The effective capacitance with series resistance increases with decreasing frequency when $R$ is small ${ }^{16}$. The decrease in $\tan \delta$ with frequency seen at high temperatures may be similar to the effect of the shunt resistance $R$. In contrast, when the temperature is low the shunt resistance $R$ can be neglected and the rise in $\tan \delta$ at high frequencies is probably due to the electrode resistance ${ }^{16}$.

The d.c. current-voltage measurements show that the specimens exhibit ohmic conduction at low electric fields but at higher fields $I \propto V^{2}$. The activation energy of $0.86 \mathrm{eV}$ and the fact that the d.c. conduction in the films is space charge limited at higher fields suggest the presence of shallow traps. A.c. conductivity is proportional to $\omega$ at low frequencies and has a square law dependence at higher frequencies. The low activation energy coupled with the square law dependence of the conductivity on frequency suggest the prevalence of an electronic hopping conduction mechanism $^{17,18}$.

The optical constants calculated from the fringe pattern of transmission spectra show that the absorption coefficient is almost zero above the fundamental absorption wavelength. Also, the refractive index is almost constant at 1.64 above $4500 \AA$. The optical band gap is found to be $3.38 \pm 0.1 \mathrm{eV}$, which is comparable with that of other kinds of insulators ${ }^{19}$.

\section{CONCLUSION}

The dielectric and optical properties of $\mathrm{Eu}_{2} \mathrm{O}_{3}$ films obtained by the vacuum evaporation of bulk material have been presented. The films possess high dielectric constant, good stability, low dissipation factor and low temperature coefficient of capacitance. The characteristics of the films are suitable for device applications. The $\mathrm{Eu}_{2} \mathrm{O}_{3}$ film described here has been successfully made use of for low voltage operation of a.c. thin film electroluminescent devices ${ }^{20}$ in metal-insulatorsemiconductor-metal structures.

\section{ACKNOWLEDGMENT}

One of the authors (M.K.J.) wishes to thank the University Grants Commission, New Delhi, for the award of a fellowship.

\section{REFERENCES}

1 U. Saxena and O. N. Srivastava, Thin Solid Films, 33 (1976) 185.

2 V. M. Koleshko and N. V. Babushkina, Thin Solid Films, 62 (1979) 1. 
3 A. Goswami and R. Ramesh Varma, Thin Solid Films, 28 (1975) 157.

4 V. S. Dharmadhirari and A. Goswami, Thin Solid Films, 87 (1982) 119.

5 H. Nakane, A. Noya, S. Kuriki and G. Matsumoto, Thin Solid Films, 59 (1979) 291.

6 R. M. Goldstein and F. W. Leonhard, Proc. Electronic Components Conf., Washington, DC, 1967, IEEE, New York, 1967, p. 312.

7 G. Hass, J. B. Ramsey and R. Thun, J. Opt. Soc. Am., 48 (1958) 324.

8 A. Goswami and A. P. Goswami, Thin Solid Films, 20 (1974) 33.

9 C. K. Campbell, Thin Solid Films, 6 (1970) 197.

10 T. Wiktorczyk and C. Wesolowska, Thin Solid Films, 71 (1980) 15.

11 F. Forlani and N. Minnaja, J. Vac. Sci. Technol., 6(1969) 518.

12 J. C. Manifacier, J. Gasiot and J. P. Fillard, J. Phys. E, 9 (1976) 1002.

13 J. Bardeen, F. J. Blott and L. M. Hall, Proc. Photoconductivity Conf., Atlantic City, NJ, Wiley, New York, 1956, p. 146.

14 K. L. Chopra, Thin Film Phenomena, McGraw-Hill, New York, 1969, pp. 189, 466-467.

15 A. A. Kornyshev, M. A. Vorotyntsev and J. Vistrup, Thin Solid Films, 75 (1981) 105.

16 A. Goswami and A. P. Goswami, Thin Solid Films, 16 (1973) 175.

17 M. Pollak and W. Geballe, Phys. Rev., 122 (1961) 1745.

18 K. Bhal and K. L. Chopra, J. Appl. Phys., 41 (1970) 2196.

19 R. C. Weast (ed.), CRC Handbook of Chemistry and Physics, CRC Press, Boca Raton, FL, 1988.

20 M. K. Jayaraj and C. P. G. Vallabhan, Bull. Electrochem., 5 (1989) 284. 